\title{
Topical Steroid Induced latrogenic Cushing Syndrome in Young Adult Age Group: A Case Report
}

\author{
Minu Kotheth George, Apollo James*, Sudhakar Reddy, Bandlamudi Yasaswini, T R Ashok kumar \\ and Thangavel Sivakumar
}

Department of Pharmacy Practice, Nandha College of Pharmacy, Erode, T.N, India.

\begin{abstract}
Background: Cushing syndrome is a systemic disorder, occurs as a result of abnormal blood level of cortisol. Prolonged use of topical corticosteroid may cause Cushing syndrome which is very rare and only a few patients have been reported to date in the literatures. Objectives: To evaluate the prompt medical care given for topical corticosteroids induced latrogenic caushing syndrome. Results: Clinical symptoms are reduced within one week of treatment with Methotrexate, Prednisone and emollients. Conclusion: Results of the study indicates that the health awareness should be created among general public to avoid the unnecessary drug induced complications.
\end{abstract}

Key words: Hypothalamo pituitory-adrenal axis suppression, latrogenic Cushing's syndrome, Psoriasis vulgaris, Topical corticosteroids.

\section{INTRODUCTION}

Psoriasis is a lifelong, chronic and immunemediated systemic disease, which affects approximately $1-3 \%$ of Caucasian population. Psoriasis may appear at any age; most often begins after puberty, but in 10\% of patients, it begins before the age of ten and $2 \%$ before the age of two. However, over $75 \%$ of patients belong to a clear sub group that develops the disease before the age of 40 with reported prevalence ranging from $2 \%$ to $4.7 \% .^{1-3}$ Psoriasisis characterized by round, erythematous, well-marginated plaques covered by characteristic grayish or silvery white scales with predilection on the scalp, elbows, knees and lumbosacral and anogenital regions. Many factors should be considered in choosing treatment for psoriasis. Topical corticosteroid is used for small localized psoriasis lesions. One of the systemic side effects is Cushing's syndrome or the physical manifestation of hypercortisolemia. This can be iatrogenic or the result of endogenous cortisol secretion, due either to an adrenal tumor or to hyper secretion of corticotrophin (adrenocorticotropic hormone) by the pituitary (Cushing disease) or by a tumor. The traditional stigmata of clinical presentations include weight gain, usually presenting as central obesity with redistribution of body fat to truncal areas and the appearance of dorsocervical and supraclavicular fatpads and the classic moon face. Plethora, easy bruising, thin skin, striae, myopathy, and muscle weakness (particularly proximal muscles) can beseen., 3,4

Submitted date :06/04/2015 Accepted date :08/06/2015

DOI: 10.5530/ijopp.8.2.8

\section{CASE REPORT}

A 19 year old male patient was admitted for psoriatic lesions (Itchy red skin rash in the arms and thighs, Acneiform eruptions over face, trunk, extremities, and generalized erythematous scaly papules/patches) which had increased during recent months. He had been diagnosed with psoriasis vulgaris 5 years before and for the past 3 years he was on treatment with topical corticosteroid clobetasol propionate $0.05 \%$ daily. Due to irregular follow-up and long-term use of topical corticosteroid, he developed moon like face, buffalo hump, early potbelly abdomen and extensive stretch marks (straie) all
Address for
correspondence:
Mr. Apollo James,
Assistant Professor,
Department of Pharmacy
Practice, Nandha College of
Pharmacy, Erode,
Tamilnadu-638052, India.
Phoneno:91-9894133961
E-mail :ajamespharma@gmail.
com 
over the body sparing legs and face. His serum cortisol levels were low $0.04 \mathrm{mcmol} / 1(0.14-0.55 \mathrm{mcmol} / \mathrm{l})$ and elevated ACTH level $76 \mathrm{pg} / \mathrm{ml}$ (normal-9-52 pg/ml). It was determined that the corticosteroids also induced obesity in this patient with $85 \mathrm{~kg}$ body weight and $162 \mathrm{~cm}$ height (BMI: $27.75 \mathrm{~kg} / \mathrm{m}^{2}$ ). His BP was 140/100 mmHg and this was considered as hypertensive. Based on these findings the patient was diagnosed with iatrogenic Cushing's syndrome caused by the prolonged use of high potency topical corticosteroids. Immediately the topical steroid was withdrawn and treatment was initiated with Methotrexate $2.5 \mathrm{mg}$ HS and a corticosteroid substitution dose of Prednisone $2.5 \mathrm{mg} /$ day to prevent adrenal insufficiency along with emollients, calcium and vitamin D supplements, After 1 month of the hospital stay, with regular monitoring and continuation of therapy, patient responded well.

\section{DISCUSSION}

Oral and topical corticosteroids have been used extensively by medical practitioners for many dermatological disorders. Patients should be informed thoroughly about the possible side effects of steroids before starting it. Otherwise it may cause severe systemic side effects including hypertension, dyslipidemia, Cushing syndrome, hypothalamo pitutuary-adrenal axis suppression, failure to thrive, glaucoma, cataract, skin atrophy, striae and predisposition to life-threatening infections..$^{5-6}$ Many factors should be considered in choosing treatment for psoriasis, such as the age of the patient, the extent and activity of the disease, site to be treated, possibleside effects, and compliance. Topical corticosteroid is used for small and localized psoriatic lesions. Prolonged use of this medication may cause local and systemic side effects which is influenced by the amount applied, frequency of application, concentration and potency of the corticosteroid, percentage of body surface covered, occlusion, long-term use, and age of patients. One of the systemic side effects is Cushing's syndrome. Inhalation, rectal administration and intra-articular injection of glucocorticoids have also been reported to be sufficient to cause clinical hypercortisolism.

\section{REFERENCES}

1. Luis Uva, et al. Mechanisms of action of topical corticosteroids in psoriasis. International Journal of Endocrinology 2012; 2012: 1-16. (doi:10.1155/2012/561018)

2. Githa Rahmayunitar, Aman B, Pulungan, Benny E, Wiryadi, Tina Wardhani Wisesar, et al. Cushing's syndrome induced by misuse of topical corticosteroids in a child with psoriasis vulgaris. Med J Indones. 2008 October-December; 17(4): 281-90.

3. JennifierVillasenor-Park, et al. Psoriasis: Evolving treatment for a complex disease. Cleveland Clinical Journal of Medicine 2012; 17(4): 79: 413-23.
Very rarely, topical corticosteroid therapy can lead to the development of Cushing's syndrome. ${ }^{1,6,7}$ Although systemic side effects of topically applied corticosteroids are much more commonly noticedin children, this phenomenon has been described in adults several times. There is some evidence that these systemic side effects are dose-dependent. ${ }^{6}$

The history and clinical findings in our patient lead us to the conclusion that he was suffering from Cushing syndrome, caused by prolonged irrational use of clobetasol propionate. Furthermore, the low cortisol level on first admission and the findings suggesting an Addisonian crisis on withdrawal of topical steroids showed that his hypothalamic-pituitary-adrenal axis must have been suppressed. To prevent adrenal suppression Prednisone 2.5 $\mathrm{mg}$ /day was given and Methotrexate $2.5 \mathrm{mg}$ HS, Vitamin D, Calcium supplements and emollients were given for managing the patient condition.

\section{CONCLUSION}

The therapeutic use of glucocorticoids for its anti-inflammatory properties including topical preparations is widely practiced for different indications including skin diseases. Apart from the irrational prescriptions, the health awareness among the general public is also lacking to the extent, that the rapid weight gain which is caused by steroids, which is basically a side effect. As a result, necessary measures should be taken by the physicians in collaboration with clinical pharmacist to make the patients aware about the need for regular follow-up during the steroid therapy to avoid drug induced complications.

\section{ACKNOWLEDGEMENT}

The Author thanks the faculties of Department of pharmacy practice, Nandha college of pharmacy for their constant support.

\section{CONFLICTS OF INTEREST}

The authors have no conflicts of interest.

4. Rachel L, Hopkins MD, Matthew C, Leinung MD. Exogenous Cushing's syndrome and Glucocorticoid Withdrawal. Endocrinal Metab Clin N Am. 2005; 93(5): 371-84.

5. West DP, Micali G. Principles of paediatric dermatological therapy. In: Harper J, Oranje A, Prose N, editors. Textbook of Paediatric Dermatology. First ed. London, Blackwell Science Ltd; 2000. P. 1731-42.

6. Ashish Tiwari, Manjusha Goel, Pankaj Pal, Poorva Gohiya. Topical-steroid-induced iatrogenic Cushing syndrome in the paediatric age group: A rare case report. Indian Journal of Endocrinology and Metabolism 2013; 17(Supplement 1): S25.

7. Abma EM. et al. Cushing's syndrome caused by topicalsteroid therapy for psoriasis Netherlands the journal of medicine. April 2002; 60(3): 148-50. 